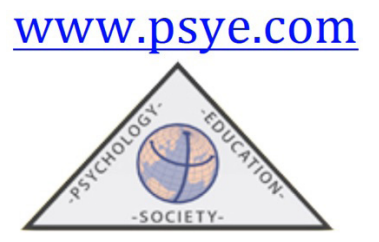

Psychology, Society, \& Education, 2020. Vol. 12(2), pp. 19-32

ISSN 2171-2085 (print) / ISSN 1989-709X (online)

Doi 10.25115/psye.v10i1.2109

\title{
Fortalezas del carácter como predictores de personalidad resistente académica y compromiso académico
}

\author{
Patricio R. ARIAS ${ }^{1}$, Felipe E. GARCÍA ${ }^{2}$, Karla $\operatorname{LOBOS}^{3}$ y Stefany C. FLORES \\ ${ }^{1}$ Instituto de Investigación Conductual (NeuroCorp), Quito, Ecuador \\ ${ }^{2}$ Facultad de Ciencias Sociales y Comunicación, Universidad Santo Tomás, Chile \\ ${ }^{3}$ Laboratorio de innovación e investigación educativa, dirección de docencia, \\ Universidad de Concepción, Concepción, Chile \\ ${ }^{4}$ Universidad Técnica del Norte, Ibarra, Ecuador
}

(Recibido el 25 de Julio de 2018, Aceptado el 13 de Abril de 2020)

\begin{abstract}
RESUMEN: Las Fortalezas del Carácter (FC) explican aspectos relevantes de la personalidad desde la psicología positiva. Las FC se las puede entender como un modelo de competencias genéricas que influyen en el desarrollo de habilidades y destrezas, y guían la puesta en práctica del conocimiento adquirido. El objetivo de esta investigación fue evaluar la influencia de estas FC sobre variables pisco-educativas como la Personalidad Resistente Académica (PRA) y el Engagement Académico (EA). Se encuestó a 263 estudiantes universitarios (20,9\% mujeres) y se les aplicó el Inventario de Virtudes y Fortalezas, la Escala de Engagement Académico y la Escala de Personalidad Resistente Académica. Se encontraron relaciones significativas entre las fortalezas evaluadas, la PRA y el EA. Las fortalezas intelectuales, emocionales y de restricción predicen la PRA y las fortalezas intelectuales y emocionales predicen el EA. En un análisis de sendero se encontró que la PRA ejerce un efecto mediador entre las FC señaladas y el EA. En conclusión, los hallazgos de este estudio permiten proponer al sistema de educación superior incluir el paradigma de la educación positiva, centrada en el desarrollo de virtudes humanas, y así, influir intencionadamente en la mejora de la retención y éxito académico de sus estudiantes.
\end{abstract}

Palabras claves: psicología positiva; rasgos; abandono universitario; educación superior; personalidad.

\section{Strengths of Character As Predictors of Academic Hardiness and Academic Engagement}

ABSTRACT: Strengths of Character (SC) explain personality from positive psychology. SC can be
understood as a model of generic competences that influence the $\mathrm{z}$ of skills and abilities, and guide the
implementation of acquired knowledge. The objective of this research was to evaluate the influence
of these SCs on pisco-educational variables such as the Academic Hardiness (AH) and the Academic
Engagement (AE). A total of 263 university students ( $20.9 \%$ female) were surveyed and the Virtues and
Strengths Inventory, the Academic Engagement Scale and the Academic Hardiness Scale were applied.
A significant correlation was found between the evaluated strengths, the AH and the AE. The intellectual, 
emotional and restriction strengths predict the $\mathrm{AH}$ and the intellectual and emotional strengths predict the AE. In a path analysis, it was found that the AH exerts a mediating effect between the designated SCs and the AE. In conclusion, the findings of this study allow the higher education system to propose to include the paradigm of positive education, focused on the development of human virtues, and thus, intentionally influence the improvement of the retention and academic success of its students.

Keywords: Positive Psychology; Traits; Leaving University; Higher Education; Personality.

Correspondencia: Felipe E. García. E-mail: felipegarciam@yahoo.es

\section{Introducción}

El presente estudio busca contribuir a una de las metas centrales de las universidades en el Siglo XXI: lograr que los estudiantes controlen su proceso de aprendizaje y adquieran competencias que les sean útiles dentro y fuera de las aulas (Park, Edmondson \& Lee, 2012), además de contribuir al abordaje del fenómeno del abandono universitario desde variables positivas que posibilitan mejores vivencias y resultados educativos en los estudiantes.

El abandono y la permanencia está siendo foco de estudio en la educación superior universitaria y actualmente se están realizando investigaciones que apuntan a modelos predictivos y explicativos que permitan procedimientos de mitigación del fenómeno y de promoción de la permanencia académica (Ayala et al., 2013; Ferreyra, Avitabile, Botero, Haimovich, \& Urzúa, 2017; Gardner, Dussán \& Montoya, 2016). Completar estudios universitarios tiene alto impacto en el proyecto de vida personal, en la familia, en la comunidad y para las instituciones, que se da a dos niveles: acciones individuales del estudiante que llevan a la persistencia en los estudios y acciones institucionales que se transforman en estrategias de retención (Esteban, Bernardo, \& Rodríguez-Muñiz; 2016; Moreta, Gaibor, \& Barrera, 2017; Tuero, Cervero, Esteban, \& Bernardo, 2018). El presente estudio se propone contribuir a ambas, intentando develar un modelo que le permita reconocer al estudiante qué actitudes académicas puede desarrollar para un mejor enfrentamiento de la experiencia universitaria y qué elementos asociados al bienestar y compromiso estudiantil pueden promover intencionadamente las instituciones de educación superior, a través de iniciativas intracurriculares, en el marco de los perfiles de egreso y/o en los programas específicos que poseen de apoyo a sus estudiantes.

En este camino, la psicología positiva propone el estudio de las características del ser humano que hacen posible su bienestar. Entre estas características está el carácter positivo llamado también virtudes y fortalezas. Peterson y Seligman (2004) proponen que el bienestar se relaciona directamente con el carácter positivo y este a su vez se relaciona directamente con rasgos positivos que luego tomaron el nombre de Fortalezas del Carácter [FC] (Cosentino, 2011). A las FC se las define como las características de personalidad similares a los rasgos moralmente valorados que contribuyen a las realizaciones que comprenden la buena vida, para uno mismo y para los demás (Ruch, Gander, Wagner, \& Giuliani, 2019).

Peterson y Seligman (2004) presentaron una taxonomía que permite identificar 24 fortalezas agrupadas originalmente en seis virtudes del carácter: a) virtud de la sabiduría y conocimiento: creatividad, curiosidad, apertura mental, amor al aprendizaje y perspectiva; b) virtud del coraje: honestidad y autenticidad, valentía, perseverancia y vitalidad; c) virtud de la humanidad: amabilidad, amor e inteligencia social; d) virtud de la justicia: equidad, liderazgo y trabajo en equipo; e) virtud

Psy, Soc, \& Educ, 2020, Vol. 12(2) 
de la templanza: perdón, modestia, prudencia y auto-regulación; f) virtud de la trascendencia: apreciación de la belleza y excelencia, gratitud, esperanza, humor, espiritualidad y religiosidad.

Por su parte, Cosentino (2011) propone cinco dimensiones que agrupan las fortalezas de carácter: a) fortalezas de restricción: prudencia, perspectiva, persistencia, integridad y autorregulación; b) fortalezas emocionales: valentía, vitalidad, esperanza, amor, humor, inteligencia social; c) fortalezas intelectuales: creatividad, curiosidad, amor por el saber, apertura; d) fortalezas interpersonales: bondad, ciudadanía, liderazgo, imparcialidad, clemencia, humildad; y, e) fortalezas teológicas: espiritualidad, gratitud y apreciación.

Si bien existen dudas sobre una estructura generalizada de las fortalezas y virtudes del carácter (Snow, 2019), se ha encontrado que estos rasgos positivos se relacionan con variables como la inteligencia emocional, el afecto positivo (Ros-Morente, Alsinet, Torrelles, Blasco, \& Jordana, 2018), la satisfacción con la vida (Yin \& Majid, 2018) y el rendimiento académico (Cosentino, Torres \& Clotet, 2009),

En el ámbito educativo el estudio de las fortalezas se ha centrado en analizar el perfil positivo de los estudiantes; por ejemplo, Jiménez, Alvarado y Puente (2013) encontraron que las virtudes más desarrolladas en estudiantes de trabajo social son la humanidad y la justicia. Así mismo, Calderón-Garrido, Forés-Miravalles, y Gustems-Carnicer (2016) encontraron que las cinco fortalezas mejor puntuadas en estudiantes universitarios con formación social fueron generosidad, igualdad, trabajo en equipo, honestidad y juicio, mientras que las fortalezas menos puntuadas fueron autorregulación, prudencia, perspectiva, creatividad y espiritualidad. Al correlacionar las fortalezas con hábitos de salud en estudiantes, se encontró una asociación positiva de la gratitud con hábitos saludables relacionados al consumo de alcohol, tabaco y otras drogas (Barrantes-Brais, Ureña-Bonilla \& Gutiérrez-Vargas, 2015). Las fortalezas también se han estudiado en la capacidad para discriminar y predecir el rendimiento académico. Por ejemplo, se ha encontrado que las FC discriminan mejor que el análisis de la personalidad entre estudiantes universitarios de alto y bajo rendimiento; las virtudes que predijeron alto rendimiento fueron sabiduría, trascendencia, coraje y templanza (Cosentino, Torres \& Clotet, 2009). En estudios más recientes se ha encontrado una correlación significativa entre la apreciación de la belleza y la excelencia, la equidad, el perdón, la honestidad, el humor, la amabilidad, el amor al aprendizaje y la humildad con el rendimiento académico en estudiantes universitarios de artes (Choudhury \& Borooah, 2017). En el estudio de Bier, Sherblom, Berkowitz, y Coulter, (2016) se pudo ver la relación entre fortalezas del carácter, como la autoeficacia, la actitud positiva, la perseverancia, la mentalidad de crecimiento, la motivación intrínseca, el cuidado intelectual y el coraje, con la educación matemática, por lo que estas fortalezas pueden ayudar a cumplir con los estándares básicos comunes de la enseñanza. También se ha podido relacionar el bienestar de los estudiantes con las fortalezas del carácter de los profesores (Yin \& Majid, 2018).

Otra variable caracterológica que estudia la psicología positiva en el ámbito educativo es la Personalidad Resistente Académica [PRA] (Carmona-Halty, Garrosa \& Moreno-Jiménez, 2017) derivada de la Personalidad Resistente o Hardiness (Kobasa, 1979). El Hardiness surgió de la comparación de diferencias individuales sobre reacciones al estrés, y se conceptualizó como una combinación de las tres actitudes: compromiso, control y desafío o reto (Kobasa, 1979; Maddi, 2006). El compromiso se refiere a la sensación profunda de involucramiento en las actividades de sus vidas (Sheard, 2009); las personas con alto nivel de compromiso se involucran en las 
actividades en lugar de retirarse, viendo el involucramiento como la mejor manera de convertir sus entornos y sus experiencias en algo interesante, importante y que vale la pena, sin importar cuán estresantes sean las actividades (Maddi, 2006.) El control refleja el deseo de las personas de continuar influyendo en los resultados que ocurren a su alrededor, en términos de lo que eligen hacer y cómo eligen responder a varios eventos sin importar cuán difícil y estresantes sean (Maddi, 2006). El desafio describe la expectativa de que la vida es impredecible y que los cambios estimularán el desarrollo personal; las personas con alto nivel de desafío creen que lo que mejora sus vidas es el crecimiento a través de aprender en lugar de comodidad y seguridad, esto les hace valorar las situaciones potencialmente estresantes como emocionantes y estimulantes en lugar de amenazantes, haciendo que las personas se sientan positivas en las fluctuaciones de la vida. (Maddi, 2006). Las sumas de estas tres actitudes proporcionan el coraje existencial, es decir, una fortaleza interna que ayuda a las personas a perseguir el futuro a pesar de su incertidumbre (Maddi, 2004),

La PRA se entiende como la puesta en práctica de las tres actitudes (compromiso, control y reto) en el área académica y en los estudios, transformando las situaciones académicas estresantes en oportunidades de crecimiento y de completación académica a pesar de la incertidumbre (Carmona-Halty et al., 2008). En estudios se ha observado que los estudiantes universitarios que han participado en un entrenamiento en personalidad resistente mostraron calificaciones más altas que el grupo control sin entrenamiento; esta mejora en el rendimiento, persistió durante un período de dos años al menos (Maddi, Harvey, Khoshaba, Fazel \& Resurrección, 2009). También se ha encontrado que la personalidad resistente tiene una influencia positiva en el compromiso o engagement en contextos organizacionales (Britt, Adler, \& Bartone, 2001; Britt \& Dickinson, 2006; Lo Bue, Taverniers, Mylle, \& Euwema, 2013), sin embargo, esta última relación no se ha estudiado en contextos educacionales.

El engagement (Maslach \& Leiter) hace referencia al estado afectivo-cognitivo persistente y que no está focalizado en un objeto o situación particular; en general es un estado mental positivo asociado con la actividad realizada (Parra \& Pérez, 2010; Salanova, Martínez, Bresó, Llorens $\&$ Grau, 2005). El engagement surge como una propuesta salugénica frente al burnout, Schaufeli et al. (2002) propone tres dimensiones que describen este concepto: a) el vigor, definido como alto nivel de energía y resistencia mental para desempeñar una labor específica; b) la dedicación, que se entiende como un alto nivel de implicación laboral; c) la absorción, tomado como un estado elevado de inmersión y concentración en la tarea que se está realizando (Schaufeli et al., 2002). El Engagement Académico [EA], a su vez, corresponde a un estado de bienestar psicológico (vigor, absorción y dedicación) y de compromiso intrínseco hacia los estudios (Parra, 2010) e implica un mayor involucramiento y satisfacción con los estudios (Parra \& Pérez, 2010). El EA ha sido relacionado positivamente con el rendimiento académico (Oriol, Mendoza, Covarrubias \& Molina, 2009; Parada \& Pérez, 2014; Parra, 2010), probablemente debido a la influencia del involucramiento sobre la planificación del aprendizaje, el aprendizaje auto-dirigido y el deseo de aprender (Spormann et al., 2015). A su vez, en un estudio desarrollado por Oriol Amutio, Mendoza, Da Costa y Miranda (2016) se encontró que fortalezas como la creatividad, la esperanza y la inspiración predecían el EA en universitarios.

En la actualidad existen estudios académicos asociados a factores cognitivos y la inteligencia, sin embargo, los factores exclusivamente intelectuales son predictores limitados del 
éxito académico a largo plazo (Castro Solano \& Casullo, 2001; Sternberg, Wagner, Williams \& Horvath, 1995). El desarrollo de las FC, la personalidad resistente frente al estrés académico y los estados positivos en estudiantes universitarios podrían ayudar a predecir con mayor exactitud el éxito académico, a prevenir el abandono escolar y promover la permanencia de estudiantes universitarios.

Una propuesta diferente, basada en la psicología positiva, es evaluar la asociación entre las Fortalezas del Carácter, la Personalidad Resistente Académica y Engagement Académico con el fin de ofrecer un modelo alternativo de competencias a desarrollar en los estudiantes universitarios para promover nuevas rutas académicas que resulten exitosas y a su vez, le sean útiles tanto en las instituciones educativas como en la vida cotidiana. En base a los estudios revisados, se propone que las FC predicen una mayor PRA y un más alto EA: a su vez, los estudios en contextos organizacionales ya citados, permiten hipotetizar que la PRA influye en el EA y, por lo tanto, actúa como mediador parcial o total entre las FC y el EA.

\section{Método}

\section{Tipo de estudio}

Esta investigación se enmarca dentro de los estudios de tipo descriptivo, correlacional, predictivo, de cohorte transversal.

\section{Participantes}

Se encuestó a 263 estudiantes de distintos niveles de la Facultad de Ingeniería de la Universidad Técnica del Norte, en Ibarra, Ecuador. Las edades fluctuaron entre 18 y 30 años $(\mathrm{M}=$ 21,$84 ; \mathrm{DE}=2,40$ ). De ellos, el 79,1\% son hombres y el 20,9\% mujeres. El 99,2 \% de los encuestados no trabajan y el $97 \%$ manifiestan ser solteros.

\section{Instrumentos}

Inventario de Virtudes y Fortalezas, IVyF (Cosentino \& Castro-Solano, 2008), evalúa de manera breve el constructo VIA original de Peterson y Seligman (2004). Está compuesto por 24 ítems bipolares en la que cada ítem describe una fortaleza y el encuestado indica en qué grado se parece más a una de dos descripciones propuestas, una con presencia de una fortaleza del carácter y la otra con ausencia. La escala presenta cinco factores: emocionales, intelectuales, interpersonales, teológicas y de restricción. Se responde en una escala tipo Likert de cinco opciones, desde 1 (soy muy parecido a la primera persona) a 5 (soy muy parecido a la segunda persona). A mayor puntaje, mayor presencia de la fortaleza. El IVyF reporta una estabilidad test-retest que oscila entre $r=0,72$ a 0,92 en sus distintas fortalezas (Cosentino \& Castro Solano, 2012). Para el presente estudio se evaluó la confiabilidad total del IVyF en la población encuestada obteniendo una adecuada consistencia interna $(\alpha=0,72)$; y la confiabilidad de sus factores reportando: Fortalezas de restricción $(\alpha=0,64)$; Fortalezas emocionales $(\alpha=0,60)$; Fortalezas intelectuales $(\alpha=0,53)$; Fortalezas interpersonales $(\alpha=0,52)$; y Fortalezas teológicas $(\alpha=0,51)$. 
Escala de Engagement Académico UWESS-9 (Schaufeli et al., 2002; traducción de Parra y Pérez, 2010). La escala presenta nueve afirmaciones (por ejemplo: "Me siento fuerte y vigoroso(a) cuando estudio o voy a clases") que se responden en una escala Likert que va de 0 (Ninguna vez) a 6 (Todos los días). En el presente estudio, esta escala obtuvo una consistencia interna de $\alpha=0,91$.

Escala de Personalidad Resistente Académica EPR-A (Carmona-Halty et al., 2017). Esta escala contiene 15 ítems (por ejemplo: "Realmente me esfuerzo e identifico con mis actividades académicas") y se contesta a través de una escala Likert que va del 1 (totalmente en desacuerdo) a 4 (totalmente de acuerdo). En el presente estudio la escala muestra una confiabilidad adecuada $(\alpha=0,84)$.

Encuesta Sociodemográfica. Se elaboró una encuesta corta que recolecta datos personales como la edad, el género, el nivel de estudio, estado laboral y estado civil.

\section{Procedimiento}

La metodología, el manejo y el procedimiento de recolección de datos fueron revisados por un docente del área de investigación de la facultad de Ingeniería de la Universidad Técnica del Norte, atendiendo a las implicaciones éticas del estudio y su alcance. Posteriormente las autoridades de la Facultad de Ingeniería de la Universidad Técnica del Norte dieron las autorizaciones necesarias para que la realización del estudio. Posteriormente se invitó a los estudiantes de la Facultad a participar, encuestando a las personas que manifestaron voluntariedad para llenar las encuestas. Por lo tanto, la selección de participantes fue por accesibilidad.

Previo a la aplicación de las encuestas se les explicó que la participación era voluntaria y sus datos serían manejados con confidencialidad y resguardados por los investigadores. Se les solicitó la firma de un consentimiento informado donde muestran su conformidad.

\section{Análisis de datos}

Luego de realizar un análisis descriptivo de las escalas, se evaluaron las correlaciones a través del coeficiente de Pearson. Para establecer la influencia de las variables predictores sobre las variables de resultado se realizaron dos regresiones lineales múltiples, una sobre la PRA y otra sobre el EA.

Finalmente se realizó un análisis de sendero utilizando el método de estimación de máxima verosimilitud. Como índices de ajuste se utilizó principalmente el $\chi^{2}$, donde se espera un valor no significativo. Además, se utilizaron el valor $\chi^{2} / \mathrm{gl}$, donde se espera un valor menor a 3 ; el CFI y TLI donde se esperan valores superiores a 0,90; el RMSEA y el SRMR donde se espera un valor inferior a 0,08 (Byrne, 2006; Kline, 2005).

El análisis de sendero muestra efectos directos, que son análogos a los puntajes beta de las regresiones lineales múltiples e indican en qué medida un cambio en una variable se relaciona con un cambio en otra variable, unidos por una flecha o path (Aron \& Aron, 2001). Los efectos indirectos son aquellos que relacionan dos variables a través de una tercera variable. La mediación se establece cuando el efecto indirecto entre dos variables tiene un valor $p$ significativo o su intervalo de confianza (IC) no pasa por el cero (Pérez, Medrano \& Sánchez-Rosas, 2013).

Para todos estos análisis se utilizó el software estadístico SPSS Statistics 23 (IBM Corp., 2013) y el programa Amos v18 de IBM (Arbuckle, 2007). 


\section{Resultados}

El análisis descriptivo de los instrumentos y las correlaciones entre las variables de estudio, se observan en la Tabla 1. En ella es posible constatar que las fortalezas de restricción, emocionales e intelectuales muestran las correlaciones más fuertes con la PRA; a su vez, las fortalezas emocionales e intelectuales muestran una relación más fuerte con EA. La correlación entre la PRA y el EA fue de un $r=0,53, p<0,001$, siendo la correlación más alta registrada entre las variables.

Tabla 1. Estadísticos descriptivos y correlaciones de las variables de estudio $(\mathrm{N}=263)$

\begin{tabular}{lccccccccccc}
\cline { 2 - 10 } & \multicolumn{4}{c}{ Descriptivos } & \multicolumn{1}{c}{ Correlaciones } \\
\cline { 2 - 11 } & Min & Max & M & DE & $\mathbf{2}$ & $\mathbf{3}$ & $\mathbf{4}$ & $\mathbf{5}$ & $\mathbf{6}$ & $\mathbf{7}$ \\
\hline 1. Fortalezas de restricción & 11 & 25 & 17,67 & 3,24 & $0,45^{* * *}$ & $0,46^{* * *}$ & $0,29^{* * *}$ & $0,18^{* * *}$ & $0,32^{* * *}$ & $0,25^{* * *}$ \\
2. Fortalezas emocionales & 11 & 30 & 20,11 & 3,75 & - & $0,39^{* * *}$ & $0,37^{* * *}$ & $0,34^{* * *}$ & $0,31^{* * *}$ & $0,31^{* * *}$ \\
3. Fortalezas intelectuales & 6 & 19 & 13,84 & 2,31 & & - & $0,22^{* * *}$ & $0,23^{* * *}$ & $0,34^{* * *}$ & $0,32^{* * *}$ \\
4. Fortalezas interpersonales & 7 & 75 & 21,02 & 5,35 & & & - & $0,19^{* *}$ & $0,23^{* * *}$ & $0,22^{* * *}$ \\
5. Fortalezas teológicas & 3 & 15 & 10,99 & 2,16 & & & & - & 0,06 & $0,14^{*}$ \\
6. Personalidad resistente académica & 29 & 60 & 46,16 & 6,75 & & & & & - & $0,53^{* * *}$ \\
7. Engagement académico & 1 & 54 & 40,37 & 9,38 & & & & & & - \\
\hline
\end{tabular}

$* \mathrm{p}<.05 ; * * \mathrm{p}<.01 ; * * * \mathrm{p}<.001$

En la tabla 2 se muestran las Regresiones Lineales Múltiples, respectivamente sobre la PRA y el EA, usando las fortalezas como predictores. En la primera de ellas se obtiene un modelo significativo, $F_{(5,257)}=11,269, \mathrm{p}<0,001$, con un $\mathrm{R}^{2}=0,18$; se observa que las fortalezas intelectuales, emocionales y de restricción ejercen una influencia significativa y directa sobre la PRA. En la segunda de ellas, también se obtiene un modelo significativo, $F_{(5,257)}=9,361, \mathrm{p}<0,001$, con un $\mathrm{R}^{2}=0,15$; las fortalezas emocionales e intelectuales ejercen una influencia significativa y directa sobre el EA.

Tabla 2. Regresiones Lineales Múltiples sobre la personalidad resistente académica y el engagement académico $(\mathrm{N}=263)$

\begin{tabular}{lcccccc} 
& \multicolumn{2}{c}{ Personalidad resistente académica } & \multicolumn{3}{c}{ Engagement académico } \\
\cline { 2 - 7 } & $\boldsymbol{\beta}$ & valor t & valor $\mathbf{p}$ & $\boldsymbol{\beta}$ & valor t & valor $\mathbf{~}$ \\
\hline (Constante) & & 8,993 & 0,000 & & 3,229 & 0,001 \\
Fortalezas de restricción & $\mathbf{0 , 1 5}$ & $\mathbf{2 , 1 5 4}$ & $\mathbf{0 , 0 3 2}$ & 0,05 & 0,746 & 0,457 \\
Fortalezas emocionales & 0,15 & 2,163 & 0,031 & 0,17 & 2,355 & 0,019 \\
Fortalezas intelectuales & 0,21 & 3,142 & 0,002 & 0,21 & 3,106 & 0,002 \\
Fortalezas interpersonales & 0,10 & 1,578 & 0,116 & 0,10 & 1,603 & 0,110 \\
Fortalezas teológicas & $-0,08$ & $-1,323$ & 0,187 & 0,01 & 0,087 & 0,930 \\
\hline
\end{tabular}


A partir de las regresiones, se procedió a probar la hipótesis del efecto mediador de la PRA entre las fortalezas y el EA. Para ello se realizó un análisis de sendero, en el cual las fortalezas de restricción, emocionales e intelectuales influían sobre la PRA y las fortalezas emocionales, intelectuales y la PRA influían sobre el EA. Sin embargo, en este modelo inicial, se observó que la influencia de las fortalezas intelectuales sobre el EA no era significativo, por lo que se procedió a eliminar este path. El modelo final, que se observa en la figura 1, obtuvo índices de ajuste significativos: $\chi^{2}=4,339, \mathrm{p}=0,114 ; \chi^{2} / \mathrm{gl}=2,17$; CFI $=0,99$; TLI $=0,96$; RMSEA $=0,067$; SRMR $=0,025$. Estos valores indican que el modelo hipotetizado ajusta con los datos del estudio.

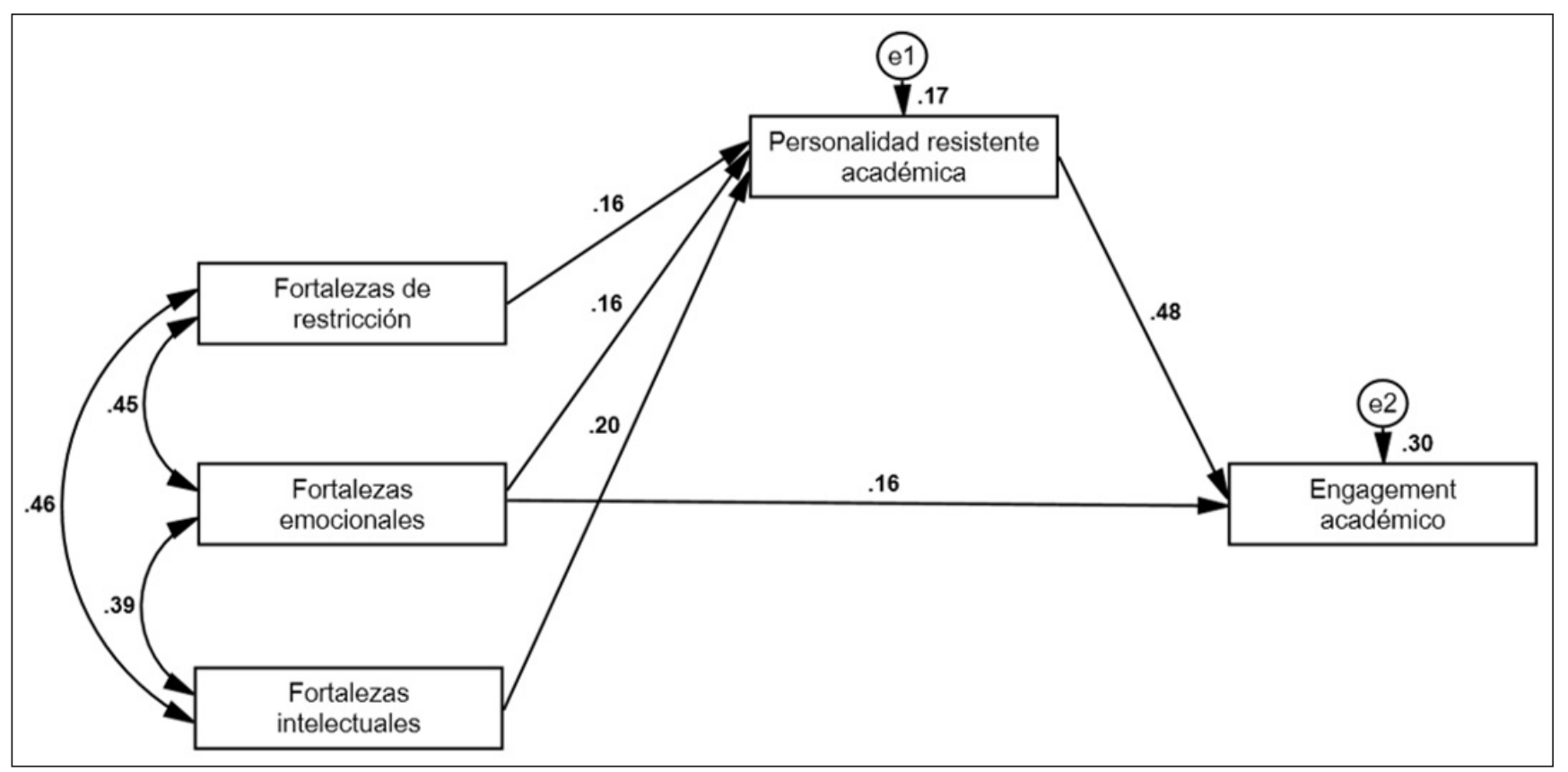

Figura 1. Modelo de sendero, los valores están estandarizados.

Al evaluar los efectos indirectos o de mediación, se observa que todos son significativos: las fortalezas de restricción sobre el EA ( $p=0,015$; IC $=0,024 / 0,135)$, fortalezas emocionales sobre EA $(p=0,027$; IC $=0,016 / 0,129)$ y fortalezas intelectuales sobre EA $(p=0,001 ; \mathrm{IC}=$ $0,040 / 0,159)$. Estos valores son coherentes con un efecto mediador total o parcial de la PRA entre las fortalezas y el EA.

\section{Discusión}

El objetivo principal de este estudio fue evaluar un modelo que permita predecir la presencia de la PRA y el EA en estudiantes universitarios. Este modelo fue analizado utilizando como variables independientes a las FC, características que permiten acercarse a una vida plena (Peterson \& Seligman, 2004).

Al realizar el análisis correlacional entre las FC, la PRA y el EA, se encontró que todas las variables se relacionan positiva y significativamente, a excepción de las fortalezas teológicas que no muestran una relación significativa con la PRA. Al parecer la resistencia al estrés, no tiene vinculación con las fortalezas teológicas, que incluye a la gratitud, la espiritualidad y la apreciación de la belleza. En cambio, las fortalezas de restricción, emocionales, intelectuales e interper- 
sonales presentan una relación importante con la PRA y el EA, coincidiendo con la apreciación de Cosentino et al. (2009) quienes mencionan que las fortalezas son adecuadas discriminadoras de desempeño académico.

La correlación entre la PRA y el EA es la más fuerte de todas las variables evaluadas, esta relación entre los factores de las dos escalas también se han encontrado en estudios previos (Carmona-Halty et al., 2017), probablemente por la coincidencia entre el compromiso con la actividad, esencial para resistir al estrés, y el EA propiamente tal. De hecho, existen numerosos estudios que indican que la personalidad resistente promueve el engagement con el trabajo (Britt, Adler, \& Bartone, 2001; Lo Bue, Taverniers, Mylle, \& Euwema, 2013) y a su vez que el estrés académico se relaciona negativamente con el EA (Bravo, 2013).

Los modelos de Regresión Lineal Múltiple encontraron que las fortalezas emocionales e intelectuales predicen la PRA y el EA, las fortalezas de restricción solo predicen una PRA. Las fortalezas interpersonales y teológicas no predicen en forma significativa la PRA ni el EA. Las fortalezas intelectuales incluyen la creatividad, la curiosidad, la apertura mental y el amor por el aprendizaje, lo que coincide con la relevancia de la creatividad sobre el EA encontrado por Oriol et al. (2016). Las fortalezas emocionales incluyen la valentía, la vitalidad, la esperanza, el amor, el humor y la inteligencia social, coincidiendo con lo encontrado en el estudio de Oriol et al. (2016) donde la esperanza también predecía EA. Finalmente, las fortalezas de restricción, que incluyen la prudencia, la perspectiva, la persistencia, la integridad y la autorregulación, las que no predicen EA, pero si PRA, lo que resulta coherente con el ejercicio de la autorregulación para el control del estrés (Park et al., 2012).

Los antecedentes teóricos disponibles (Cosentino et al., 2009), los antecedentes previos respecto a la influencia de la personalidad resistente sobre el engagement (Britt et al., 2001; Lo Bue et al., 2013) y los antecedentes aportados por las regresiones, posibilitaron la construcción de un modelo de sendero con suficiente sustento teórico y empírico. Este modelo mostró ajustarse adecuadamente a los datos disponibles, y su principal propuesta es que la PRA ejerce un rol mediador entre las FC y el EA. Este resultado permite prever que una personalidad capaz de resistir el estrés relacionado al ámbito académico resulta esencial para que las FC, muchas de ellas desarrolladas en forma previa al ingreso de un estudiante a la educación superior, puedan influir sobre un mayor EA, a su vez predecir la planificación del aprendizaje (Spormann et al., 2015) y el buen rendimiento (Parra, 2010).

En consecuencia, estos resultados contribuyen con evidencia para promover actitudes de compromiso, control y desafío, incentivando a que los estudiantes se involucren en las actividades en lugar de evitarlas o retirarse, buscando influir en sus procesos de aprendizaje, con mayor control de las situaciones que le afectan y con una actitud constructiva ante un mundo cambiante, más que en el confort de lo ya conocido y estable. De particular relevancia, es que estos resultados tributan a una población altamente vulnerable, pues las carreras de ingeniería son de las que tienen una más alta tasa de abandono y no titulación en el Ecuador (Bazantes, Ruiz, \& Álvarez, 2017; Ramírez, 2016; Sinchi \& Gómez, 2018). Ayudar a resistir el estrés, sobre todo los primeros años de educación superior, y contribuir a un mayor bienestar y compromiso de los estudiantes universitarios, son preocupaciones que deben estar presentes a la hora de desarrollar los planes y programas de estudio, así como en las políticas de retención que las instituciones de educación superior se propongan. 
Entre las limitaciones de este estudio podemos mencionar su naturaleza transversal, pues si bien se presenta un análisis de sendero, que hipotetiza relaciones causales, no es posible en este caso hablar de causalidad pues las variables fueron todas medidas en un único momento. Esto plantea la necesidad de realizar estudios longitudinales que midan estas variables al iniciar la vida académica universitaria y luego seguir su rendimiento a lo largo de su proceso de formación. Una segunda limitación es la selección de la muestra, pues en el presente caso fue por accesibilidad, lo que no asegura que la muestra sea representativa de la población. De ese modo, los alcances del estudio están limitados a la población específica de estudiantes que fueron encuestados y su generalización a otras poblaciones tiene carácter hipotético. Una tercera limitación es la ausencia de datos sobre el rendimiento de los estudiantes, pues debido al momento en que se realizó el estudio no se contaban con datos acerca del rendimiento final por asignatura. El dato del rendimiento académico permitiría construir modelos predictivos más complejos y de mayor poder explicativo a fin de predecir el desempeño, por ejemplo, la realización de estudios longitudinales y análisis de crecimiento latente, que permitirían evaluar el poder predictivo de las variables incluidas en este estudio además de controlar otras variables como el nivel educativo, asignatura y profesor, entre otras.

Otra propuesta para estudios futuros sería evaluar estos mismos factores en estudiantes de secundaria, ya en la universidad la intervención tiende a ser más reparatoria. También se sugiere estudiar a alumnos de educación técnica profesional, en donde se agrupan los sectores de jóvenes más desaventajados en los ámbitos académicos, lo que dificulta a las instituciones hacerse cargo de las diferencias de entrada de sus estudiantes. Este enfoque positivo puede ayudar a promover el empoderamiento y la superación de las dificultades de entrada de parte de los propios estudiantes, incentivándolos a asumir un rol más activo.

Estos hallazgos resultan novedosos pues permiten la transferencia de un conocimiento ampliamente utilizado en áreas de la salud, clínica o laboral, al ámbito académico, complementando los estudios tradicionales centrados en factores cognitivos, de desempeño en niveles educativos previos, o sociodemográficos, con estudios basados en la psicología positiva. A partir de los hallazgos de este estudio el sistema de educación superior podría incluir el paradigma de la educación positiva, centrada en el desarrollo de virtudes humanas, y así influir intencionadamente en el éxito académico de sus estudiantes.

\section{Referencias bibliográficas}

Arbuckle, J. L. (2007). Amos ${ }^{\mathrm{TM}} 18$ User's Guide. Crawfordville, USA: Amos Development Corporation

Aron, A. \& Aron, E. (2001). Estadística para psicología. Buenos Aires, Argentina: Pearson Education

Ayala, M.C., Castro, C., Fernández, V., Gallardo, G., Jouannet, C. \& Moreno, K. (2013). Inclusión, acogida y apoyo hacia los estudiantes desde las instituciones de educación superior. Santiago de Chile: AEQUALIS.

Barrantes-Brais, K., Ureña Bonilla, P. \& Gutiérrez Vargas, J. C. (2015). Fortalezas Personales y Hábitos de Salud en Estudiantes Universitarios de Ciencias del Movimiento Humano. Revista MHSalud, 11(2), 20-36. doi: 10.15359/mhs.11-2.2

(C) Psy, Soc, \& Educ, 2020, Vol. 12(2) 
Bazantes, Z. P., Ruiz, M. L. \& Álvarez, M. L. (2017). Deserción estudiantil universitaria en Ecuador y su influencia en la calidad del egresado. Revista Magazine de las Ciencias, 1(4), 65-70. Disponible en: https://revistas.utb.edu.ec/index.php/magazine/article/view/183

Bier, M. C., Sherblom, S. A., Berkowitz, M. W., \& Coulter, B. (2016). The ways character strengths support k-8 mathematics and the common core state standards. Journal of Character Education, 12(1), 35-53. Disponible en: https://eric.ed.gov/?id=EJ1151719

Bravo, L. (2013). Predictores de engagement académico en estudiantes de odontología. RECS: Revista Educación en Ciencias de la Salud, 10(2), 86-95. Disponible en: www2.udec.cl/ ofem/recs/anteriores/vol1022013/artinv10213b.htm

Britt, T. W., \& Dickinson, J. M. (2006). Morale during military operations: A positive psychology approach. In T. W. Britt, C. A. Castro, \& A. B. Adler (Eds.), Military life: The psychology of service in peace and combat: Vol. 1. Military performance (pp. 157-184). Westport, CT: Praeger Security International.

Britt, T. W., Adler, A. B., \& Bartone, P. T. (2001). Deriving benefits from stressful events: The role of engagement in meaningful work and hardiness. Journal of Occupational Health Psychology, 6(1), 53-63. doi: 10.1037//1076-8998.6.1.53

Byrne, B. (2006). Structural equation modeling with Mplus. New York, NY: Routledge.

Calderón-Garrido, D., Forés-Miravalles, A., Gustems-Carnicer, J. (2016). Aproximación a las fortalezas de carácter en los estudiantes de Educación Social. Perfil de una muestra. REIRE. Revista d'Innovació i Recerca en Educació, 9(2), 48-64. doi: 10.1344/reire2016.9.2924

Carmona-Halty, M., Garrosa, E. \& Moreno-Jiménez, B. (2017). Análisis psicométrico de la Escala de Personalidad Resistente (EPR) adaptada a estudiantes universitarios chilenos. Interciencia, 42(5), 286-292. Disponoble en: https://www.redalyc.org/pdf/339/33952810004.pdf

Castro Solano, A. \& Casullo, M. M. (2001). Rasgos de personalidad, bienestar psicológico y rendimiento académico en adolescentes argentinos. Interdisciplinaria, Revista de Psicología $y$ Ciencias Afines, 18(1), 65-85. Disponible en: www.redalyc.org/pdf/180/18011326003.pdf

Choudhury, S. A., \& Borooah, I. P. (2017). Character strengths and academic achievements of undergraduate college students of Guwahati, Assam. Space and Culture, India, 5(1), 49-64. doi: $10.20896 /$ saci.v\%vi\%i.242

Cosentino, A. C. \& Castro Solano, A. (2008). Inventario de virtudes y fortalezas. Manuscrito no publicado.

Cosentino, A. C. \& Castro Solano, A. (2012). Character strengths: A study of Argentinean soldiers. The Spanish Journal of Psychology, 15(1), 199-215. doi: 10.5209/rev_SJOP.2012. v15.n1.37310

Cosentino, A. C. (2011). Fortalezas del carácter en militares argentinos. (Tesis doctoral). Universidad de Palermo, Buenos Aires, Argentina.

Cosentino, A. C., Torres, J. A. \& Clotet, C. I. (2009, agosto). El carácter moral predice el rendimiento académico. Ponencia presentada al Primer Congreso Internacional de Investigación y Práctica Profesional en Psicología, Facultad de Psicología, Universidad de Buenos Aires, Buenos Aires.

Esteban, M., Bernardo, A., \& Rodríguez-Muñiz, L.J. (2016). Permanencia en la universidad: la importancia de un buen comienzo. Aula Abierta, 44, 1-6. doi. 10.1016/j.aula.2015.04.001 
Ferreyra, M., Avitabile, C., Botero J., Haimovich, F. \& Urzúa, S. (2017). Momento decisivo. La educación superior en América Latina y el Caribe. Washington, DC: Banco Mundial.

Gardner, L., Dussán, C., \& Montoya, D. (2016). Aproximación causal al estudio de la deserción en la Universidad de Caldas. Periodo 2012-2014. Revista Colombiana de Educación, 70, 319-340. doi: 10.17227/01203916.70rce319.340

IBM Corp (2013). IBM SPSS Statistics for Windows, Version 22.0. Armonk, NY: IBM corp. Jiménez Rodríguez, V., Alvarado Izquierdo, J. M., \& Puente Ferreras, A. (2013). Una aproximación al Trabajo Social desde la óptica de la Psicología positiva (virtudes y fortalezas). Cuadernos de Trabajo Social, 26(2), 397-407. doi: 10.5209/rev_cuts.2013.v26.n2.40915

Kline, R. B. (2005). Principles and practice of Structural Equation Modeling (2nd ed.). New York: Guilford.

Kobasa, S. C. (1979). Stressful life events, personality, and health: An inquiry into hardiness. Journal of Personality and Social Psychology, 37(1), 1-11. doi: 10.1037/00223514.37.1.1

Lo Bue, S., Taverniers, J., Mylle, J., \& Euwema, M. (2013). Hardiness promotes work engagement, prevents burnout, and moderates their relationship. Military Psychology, 25(2), 105-115. doi: $10.1037 / \mathrm{h} 0094952$

Maddi, S. R. (2004). Hardiness: An operationalization of existential courage. Journal of humanistic psychology, 44(3), 279-298. doi: 10.1177/0022167804266101

Maddi, S. R. (2006). Hardiness: The courage to grow from stresses. The Journal of Positive Psychology, 1(3), 160-168. doi: 10.1080/17439760600619609

Maddi, S. R., Harvey, R. H., Khoshaba, D. M., Fazel, M. \& Resurrección, N. (2009). Hardiness training facilitates performance in college. Journal of Positive Psychology, 4(6), 566-577. doi: 10.1080/17439760903157133

Maslach, C. \& Leiter, M. (1997). The truth about burnout. San Francisco, CA: Jossey Bass.

Moreta, R., Gaibor, I., \& Barrera, L. (2017). El bienestar psicológico y la satisfacción con la vida como predictores del bienestar social en una muestra de universitarios ecuatorianos. Salud \& Sociedad, 8 (2), 172-184. doi: 10.22199/S07187475.2017.0002.00005

Oriol, X., Amutio, A., Mendoza, M., Da Costa, S., \& Miranda, R. (2016). Emotional creativity as predictor of intrinsic motivation and academic engagement in university students: The mediating role of positive emotions. Frontiers in Psychology, 7, 1-9 doi: 10.3389/ fpsyg.2016.01243

Oriol, X., Mendoza, M., Covarrubias, C. G., \& Molina, V. (2017). Positive emotions, autonomy support and academic performance of university students: the mediating role of academic engagement and self-efficacy. Revista de Psicodidáctica, 22(1), 45-53. doi: 10.1387/ revpsicodidact. 14280

Parada, M., \& Pérez, C. (2014). Relación del engagement académico con características académicas y socioafectivas en estudiantes de odontología. Educación Médica Superior, 28(2), 199-215. Disponible en: http://scielo.sld.cu/pdf/ems/v28n2/ems03214.pdf

Park, C.L., Edmondson, D., \& Lee, J. (2012). Development of self-regulation abilities as predictors of psychological adjustment across the first year of college. Journal of Adult Development, 19, 40-49. doi:10.1007/s10804-011-9133-z

(C) Psy, Soc, \& Educ, 2020, Vol. 12(2) 
Parra, P. \& Pérez, C. (2010). Propiedades psicométricas de la escala de compromiso académico, UWES-S (versión abreviada), en estudiantes de psicología. RECS: Revista de Educación en Ciencias de la Salud, 7(2) 128-133. Disponible en: www2.udec.cl/ofem/recs/anteriores/ vol722010/artinv7210c.pdf

Parra, P. (2010). Relación entre el nivel de engagement y el rendimiento académico teórico/ práctico. RECS: Revista de Educación en Ciencias de la Salud, 7(1), 57-63. Disponible en: http://www2.udec.cl/ofem/recs/anteriores/vol712010/revbib71a.pdf

Pérez, E., Medrano, L. A., \& Sánchez Rosas, J. (2013). El Path Analysis: Conceptos básicos y ejemplos de aplicación. Revista Argentina de Ciencias del Comportamiento, 5(1), 52-66

Peterson, C., \& Seligman, M. E. (2004). Character strengths and virtues: A handbook and classification (Vol. 1). New York, NY: Oxford University Press.

Peterson, C., \& Seligman, M. E. P. (2003). Character Strengths Before and After September 11. Psychological Science, 14(4), 381-384. doi: 10.1111/1467-9280.24482

Ramírez, R. (2016). Universidad urgente para una sociedad emancipada. Quito: IESALC.

Ros-Morente, A., Alsinet, C., Torrelles, C., Blasco, A., \& Jordana, N. (2018). An examination of the relationship between emotional intelligence, positive affect and character strengths and virtues. Anales de Psicología, 34(1), 63-67. doi: 10.6018/analesps.34.1.262891

Ruch, W., Gander, F., Wagner, L., \& Giuliani, F. (2019). The structure of character: On the relationships between character strengths and virtues. The Journal of Positive Psychology, 1-13. doi: 10.1080/17439760.2019.1689418

Ruch, W., Proyer, R. T., Harzer, C., Park, N., Peterson, C., \& Seligman, M. E. P. (2010). Values in Action Inventory of Strengths (VIA-IS). Journal of Individual Differences, 31(3), 138-149. doi: 10.1027/1614-0001/a000022

Salanova, M., Martínez, I., Bresó, E., Llorens, S., \& Grau, R. (2005). Bienestar psicológico en estudiantes universitarios: Facilitadores y obstaculizadores del desempeño académico. Anales de Psicología, 21(1), 170-180. doi: 10.6018/analesps

Schaufeli, W., Martínez, I., Marques-Pinto, A., Salanova, M., \& Bakker, A. B. (2002). Burnout and engagement in university students: A cross-national study. Journal of Cross-Cultural Psychology, 33(5), 464-481. doi: 10.1177/0022022102033005003

Seligman, M. E. P., \& Csikszentmihalyi, M. (2000). Positive psychology: An introduction. American Psychologist, 55, 5-14. doi:10.1037/0003-066X.55.1.5

Sheard, M. (2009) Hardiness commitment, gender, and age differentiate university academic performance. British Journal of Educational Psychology, 79(1), 189-204. doi: 10.1348/000709908X304406

Shimai, S., Otake, K., Park, N., Peterson, C. y Seligman, M. E. P. (2006). Convergence of character strengths in American and Japanese young adults. Journal of Happiness Studies, 7(3), 311- 322. doi: 10.1007/s10902-005-3647-7

Sinchi, E., \& Gómez, G. (2018). Acceso y deserción en las universidades. Alternativas de financiamiento. Alteridad, 13(2), 274-287. doi: 10.17163/alt.v13n2.2018.10

Snow, N. E. (2019). Positive psychology, the classification of character strengths and virtues, and issues of measurement. The Journal of Positive Psychology, 14(1), 20-31. doi: $10.1080 / 17439760.2018 .1528376$ 
Spormann R, C., Pérez V, C., Fasce H, E., Ortega B, J., Bastías V, N., Bustamante D, C., \& Ibáñez G, P. (2015). Predictores afectivos y académicos del aprendizaje autodirigido en estudiantes de medicina. Revista Médica de Chile, 143(3), 374-382. doi: 10.4067/s003498872015000300013

Sternberg, R., Wagner, R., Williams, W. \& Horvath, J. (1995). Testing common sense. American Psychologist, 50(11), 912-927. doi: 10.1037/0003-066X.50.11.912

Tuero, E., Cervero, A., Esteban, M., \& Bernardo, A. (2018). ¿Por qué abandonan los alumnos universitarios? variables de influencia en el planteamiento y consolidación del abandono. Educación XXI, 21(2), 131-154. doi: 10.5944/educxx1.20066

Yin, L. C., \& Majid, R. A. (2018). The goodness of character strengths in education. International Journal of Academic Research in Business and Social Sciences, 8(6), 1237-1251. doi: 10.6007/ijarbss/v8-i6/4512 